\title{
THE
}

UNIVERSITY

University of Rhode Island

OF RHODE ISLAND

DigitalCommons@URI

$2-5-2003$

\section{Surface Roughness and Effective Stick-Slip Motion}

I. V. Ponomarev

A. E. Meyerovich

University of Rhode Island, sfo101@uri.edu

Follow this and additional works at: https://digitalcommons.uri.edu/phys_facpubs

Terms of Use

All rights reserved under copyright.

\section{Citation/Publisher Attribution}

Ponomarev, I. V., \& Meyerovich, A. E. (2003). Surface Roughness and Effective Stick-Slip Motion. Phys.

Rev. E, 67, 026302. doi: 10.1103/PhysRevE.67.026302

Available at: http://dx.doi.org/10.1103/PhysRevE.67.026302

This Article is brought to you for free and open access by the Physics at DigitalCommons@URI. It has been accepted for inclusion in Physics Faculty Publications by an authorized administrator of DigitalCommons@URI. For more information, please contact digitalcommons-group@uri.edu. 


\title{
Surface roughness and effective stick-slip motion
}

\author{
I. V. Ponomarev* and A. E. Meyerovich ${ }^{\dagger}$ \\ Department of Physics, University of Rhode Island, Kingston, Rhode Island 02881-0817
}

(Received 17 May 2002; published 5 February 2003)

\begin{abstract}
The effect of random surface roughness on hydrodynamics of viscous incompressible liquid is discussed. When the hydrodynamic decay length (the viscous wave penetration depth) is larger than the correlation radius (size) of random surface inhomogeneities, it is possible to replace a random rough surface by effective stick-slip boundary conditions on a flat surface with two constants: the stick-slip length and the renormalization of viscosity near the boundary. The stick-slip length and the renormalization coefficient are expressed explicitly via the correlation function of random surface inhomogeneities. The stick-slip length is always negative and the effective change of viscosity near the surface is positive signifying the effective average hampering of the hydrodynamic flows by the rough surface (stick rather than slip motion). A simple hydrodynamic model illustrates general hydrodynamic results. The effective boundary parameters are analyzed numerically for Gaussian, power-law and exponentially decaying correlators with various indices. The maximum on the frequency dependence of the dissipation allows one to extract the correlation radius (characteristic size) of the surface inhomogeneities directly from, for example, experiments with torsional quartz oscillators.
\end{abstract}

DOI: 10.1103/PhysRevE.67.026302 PACS number(s): 47.10.+g, 68.08. $-\mathrm{p}, 46.65 .+\mathrm{g}, 81.40 . \mathrm{Pq}$

\section{INTRODUCTION}

Progress in microtechnology and nanotechnology requires a better understanding of boundary effects. For hydrodynamic microflows, this means a better understanding of stick-slip motion near solid walls and, in particular, information on the dependence of the slip (or stick) length on the properties of the walls. Despite the fact that similar issues were first raised more than a hundred years ago [1-3], the slip length remains one of the least known transport coefficients.

Traditionally, the most detailed information on boundary slip is available for rarefied classical gases [4-7] in application to vacuum technology, high altitude flights, and space research. More recently [8-15], liquid ${ }^{3} \mathrm{He}$ has become an important source of information on surface slip. This is not surprising since, in contrast to classical gases, one can easily vary the quasiparticle mean free path in ${ }^{3} \mathrm{He}$ by changing temperature, thus allowing experiments in a wide range of Knudsen numbers.

The miniaturization of experimental systems renewed interest to slip effects in normal liquids with short free paths, in which the slip length is in nanometer range. This slip length, though very small, is, nevertheless, extremely important for hydrodynamic flows in nanochannels and microchannels, lubrication, etc. Modern experimental techniques, including the atomic force microscopy [16-19], experiments with powder or self-assembled monolayers [20-22], optical methods [23], quartz crystal microbalance [24], etc., revealed the importance of (partial) surface slip for hydrodynamic flows in narrow channels and layers between solid walls.

The conventional gas and hydrodynamic theories assume

\footnotetext{
*Electronic address: ilya@qc.edu; present address: Department of Physics, Queens College of CUNY, Kissena Blvd. Flushing, NY 11367.

${ }^{\dagger}$ Electronic address: Alexander_Meyerovich@uri.edu
}

that there are two main sources of noticeable boundary slip: large bulk mean free path, as in rarefied gases [7] or ${ }^{3} \mathrm{He}$ (for review, see Ref. [13], and references therein) or, in dense liquids with atomic-size free path, peculiarities of molecular interaction with the wall (for review, see Ref. [25], and references therein) such as, for example, the molecular slip or formation of a gas-rich layer near the hydrophobic surface. In the former case, the slip length is assumed to be proportional to the large bulk mean free path $\mathcal{L}_{b}, \mathcal{L}_{s l}=\alpha \mathcal{L}_{b}$. In the latter case, the boundary slip is associated with molecular interaction with the wall and becomes noticeable only in special cases such as hydrophobic walls, electrolytes, large molecules, etc.

The change in the flow patterns caused by the roughness of the walls, though often acknowledged as the third potential source of stick-slip motion near the walls, is usually disregarded. The reason is the complexity of the flows near rough walls and the resulting difficulty in formulating general quantitative conclusions. It is understood that the boundary corrugation changes the liquid and gas flows in a wall layer with a thickness comparable to the parameters of the corrugation. However, this change is considered to be not sufficiently large for flows in wider channels to merit a detailed theoretical analysis of this complicated hydrodynamic issue. Besides, it is not always easy to treat random wall roughness in a consistent quantitative way. In the case of dilute gases, it seems reasonable to ignore small-scale surface inhomogeneities on the scale smaller than the large bulk mean free path $\mathcal{L}_{b}$. In the case of dense liquids, the theory [26-28] indicates that the microscale surface roughness hinders the flow near the wall and can, by itself, become the origin of the no-slip boundary condition; apart from this general conclusion, the details of the roughness-induced changes in the flow are assumed to be case specific. On the other hand, the numerical methods [29,30] are not sufficiently well developed so that to include simultaneously the change in molecular forces near the liquid-solid interface and the complicated geometry (random roughness) of the surface (for 
some recent progress in this direction, see Ref. [31]).

Experimentally microflows and nanoflows can exhibit, depending on the experimental setup and the wetting properties of the liquid-solid interface, full range of slip properties from complete slippage to partial slip to no slip to stick ("freezing"). So far, there is no systematic analysis of the additional effects introduced by surface roughness on the scale comparable to the bulk-driven stick-slip length. From this point of view, the situation is not yet satisfactory especially if one takes into account current interest to microflows and nanoflows, for which the roughness-driven change of flow patterns close to the walls are not negligible. For nanoflows, this deficiency of the existing theory could become inexcusable.

Recent analysis of a slip near a model surface with periodic irregularities [32] demonstrated that the slip length $\mathcal{L}_{s l}$ in dilute gases contains not only the bulk component $\alpha \mathcal{L}_{b}$, but also the contribution from the averaged surface curvature, $\mathcal{L}_{s l}^{-1}=\alpha^{-1} \mathcal{L}_{b}^{-1}-R^{-1}$. An application of the corresponding boundary condition to several types of curved walls [33] resulted in an interesting expression for an effective slip length which could, under certain circumstances, be equivalent to large-scale surface roughness. However, these results $[32,33]$ were obtained for few special types of regular surface inhomogeneities only. In the case of microscale and nanoscale defects, it is more realistically to suggest that surfaces have random corrugation. What is more, in some cases, especially in the hydrodynamic limit $\mathcal{L}_{b} \rightarrow 0$, it is not clear how to use the effective boundary parameters of Refs. $[32,33]$.

Below, we address the issue of hydrodynamic flows near rough walls with small-scale random inhomogeneities. Since the hydrodynamic calculations near inhomogeneous walls are extremely complicated [30], it is highly desirable to map this problem onto the problem with some effective boundary condition on an ideal flat wall. This general effective boundary condition should contain information about geometrical and statistical properties of the real corrugated surface and ensure a proper behavior of physical variables. The derivation of this simple effective boundary condition is the main objective of the paper. We will show that this boundary condition contains two effective parameters: stick-slip length and renormalized viscosity. We will also demonstrate that the results for attenuation in torsional oscillator experiments can provide valuable information about the statistical type of surface inhomogeneities and give the values of the main geometrical parameters of the surface roughness.

In this paper, we are interested exclusively in the contribution to the effective surface stick-slip coming from the surface roughness and ignore the slip terms originating from the wetting-related processes. Therefore, we start from a traditional no-slip boundary condition on the wall with random inhomogeneities. Though in Sec. V of the paper, we discuss how to use this effective stick-slip length in conjunction with other sources of the surface slip, this broader issue requires separate analysis.

In the following section, we present the main hydrodynamic equations and find a general expression for the stream function in systems with random rough walls (the details of

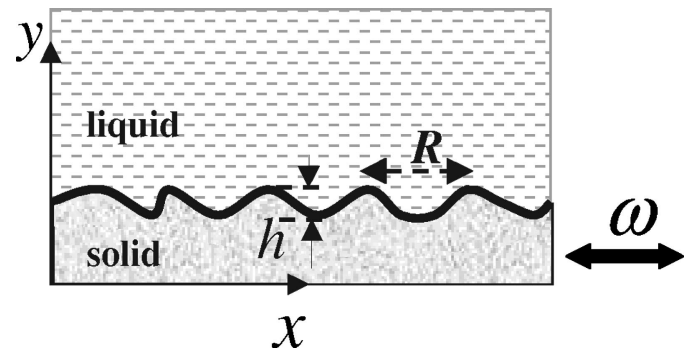

FIG. 1. General geometry of the model.

the derivation are given in Appendix A). Comparison of this hydrodynamic result with the expressions for the stick-slip motion near flat walls allows us to get the expression for the effective stick-slip parameters in Sec. III. For clarification of the physical meaning of the parameters in the somewhat unexpected effective boundary condition, we present a simple hydrodynamic model for a boundary layer in Appendix B. Section IV contains analytical and numerical results for the surfaces with various statistical types of inhomogeneities. In Sec. V, we present the conclusions and discuss further steps that can broaden the impact of our results.

\section{HYDRODYNAMIC FLOWS ALONG ROUGH WALLS}

To determine an effective slip or stick length, one has to solve an appropriate hydrodynamic problem with a boundary condition on a random rough wall and to compare the results with those for a similar problem with a slip boundary condition on a smooth wall. Several "typical" hydrodynamic problems [34] have been generalized recently in order to cover boundaries with slight roughness [35-38]. For our purposes, the most appropriate problem is the problem of hydrodynamic flows excited by tangential oscillations of a rough wall. The advantages are the convenience of the experimental setup with a standard transverse oscillator, a choice of several observables such as hydrodynamic velocity and two components of the shear impedance, and the presence of an extra variable-frequency $\omega$-which allows one to vary the ratio of the hydrodynamic decay length to the size of wall inhomogeneities. Since this problem has already been studied in Ref. [35], though by a different method, we will only briefly outline our hydrodynamic formalism in Appendix A and present some additional results.

We consider semi-infinite viscous fluid restricted by a rough solid wall. For simplicity, roughness is assumed to be one-dimensional and have a profile described by a random function $Y=\Xi(X)$ with the zero mean value, $\langle\Xi(X)\rangle=0$. The wall is homogeneous in the $Z$ direction (see Fig. 1). This inhomogeneous surface is characterized by two length parameters - the average amplitude $h$ and correlation radius (size) $R$ of surface inhomogeneities. We consider the case of slight roughness,

$$
\epsilon=h / R \ll 1 .
$$

In other situations, any general description of hydrodynamic flows near rough walls is virtually impossible.

The wall oscillates in $X$-direction with the velocity 


$$
\mathbf{U}(t)=\mathbf{e}_{x} u_{0} \cos (\omega t) .
$$

The hydrodynamic boundary condition is the condition of zero velocity $\mathbf{V}$ on the wall in the reference frame, in which the wall is at rest,

$$
\mathbf{V}\left(X-\int \mathbf{U}(t) d t, Y=\Xi\left(X-\int \mathbf{U}(t) d t\right)\right)=0 .
$$

Two important hydrodynamic length scales are the decay length (or viscous wave penetration depth) $\delta$ and the amplitude of the boundary oscillations, $a$,

$$
\delta=\sqrt{2 \nu / \omega}, \quad a=u_{0} / \omega,
$$

where $\nu=\eta / \rho$ is the kinematic viscosity.

It is convenient to choose $h, R$, and the amplitude of the wall velocity $u_{0}$ as the scaling parameters and introduce dimensionless variables as

$$
\mathbf{v}=\mathbf{V} / u_{0}, \quad x=X / R, \quad y=Y / R, \quad \xi(x)=\Xi(X / R) / h .
$$

When the fluctuations of $\xi(x)$ are statistically independent and the higher momenta can be expressed through the second one, the random surface roughness is actually described not by the unknown random function $\xi(x)$ with the zero average, but by the correlation function $\zeta(x)$,

$$
\begin{gathered}
\zeta(x) \equiv\left\langle\xi\left(x_{1}\right) \xi\left(x_{1}+x\right)\right\rangle=\frac{1}{A} \int_{-\infty}^{\infty} \xi\left(x_{1}\right) \xi\left(x_{1}+x\right) d x_{1}, \\
\left\langle\xi\left(k_{x}\right) \xi\left(k_{x}^{\prime}\right)\right\rangle=2 \pi \delta\left(k_{x}+k_{x}^{\prime}\right) \zeta\left(k_{x}\right),
\end{gathered}
$$

where $A$ is dimensionless flat surface area of the wall. Experimentally, the correlation functions $\zeta(x)$ [or its Fourier image, also known as the power spectrum, $\left.\zeta\left(k_{x}\right)\right]$ can exhibit different types of long-range behavior and assume various forms [39]. Particular examples of the surface correlators are analyzed in Sec. IV. Note that in our dimensionless notations (5), the correlation radius of surface inhomogeneities is equal to 1 .

The liquid is considered incompressible, $\operatorname{div} \mathbf{v}=0$. In variables (5), the dimensionless Navier-Stokes equation can be written as

$$
\frac{1}{\omega_{0}} \frac{\partial \operatorname{curl} \mathbf{v}}{\partial t}-\nabla^{2} \operatorname{curl} \mathbf{v}=\operatorname{Re}[(\operatorname{curl} \mathbf{v} \boldsymbol{\nabla}) \mathbf{v}-(\mathbf{v} \boldsymbol{\nabla}) \operatorname{curl} \mathbf{v}],
$$

where the characteristic frequency $\omega_{0}$ and the Reynolds number Re are

$$
\omega_{0}=\frac{\nu}{R^{2}}, \quad \operatorname{Re}=\frac{u_{0} R}{\nu} \equiv \frac{a}{R} \frac{\omega}{\omega_{0}}
$$

(the inverse frequency parameter $\omega_{0}^{-1}$ is often called the diffusion time of vorticity). Since the first term in Eq. (7) has an order of $\left(\omega / \omega_{0}\right)$ curl $\mathbf{v}$, the hydrodynamic flows are characterized by the dimensionless parameter

$$
\Lambda=\sqrt{\omega / \omega_{0}}=\sqrt{2} R / \delta,
$$

which describes the ratio of the size of inhomogeneities $R$ to the hydrodynamic decay length $\delta$. Two dimensionless parameters, $\epsilon$ and $\Lambda$, are the main parameters of the problem.

Below, we consider the linearized Navier-Stokes equation without the nonlinear term on the right-hand side (RHS) of Eq. (7). For small frequencies, $\omega / \omega_{0} \ll 1$, this linearization is justified for very small Reynolds numbers $\operatorname{Re} \ll 1$. In the opposite limit of high frequencies, $\omega / \omega_{0} \gg 1$, this requires smallness of the amplitude of oscillations $a$ in comparison with the tangential size of surface inhomogeneities $R$ at arbitrary Reynolds numbers [34] Re, $a / R \ll 1$. The linearized Eq. (7) for curl $\mathbf{v}$ can be, as usual, rewritten as the fourthorder differential equation for the scalar stream function $\psi(x, y)$,

$$
v_{x}=\frac{\partial \psi}{\partial y}, \quad v_{y}=-\frac{\partial \psi}{\partial x} .
$$

In our problem, all hydrodynamic variables contain harmonic time dependence. After the transformation to the coordinate frame oscillating with the wall, the hydrodynamic equations and boundary conditions for the stream function acquire the form

$$
\begin{gathered}
-i \Lambda^{2} \nabla^{2} \psi-\nabla^{4} \psi=0, \\
\frac{\partial \psi(x, \epsilon \xi(x))}{\partial y}=1, \quad \frac{\partial \psi(x, \epsilon \xi(x))}{\partial x}=0, \\
\psi(x, \infty)=\text { const. }
\end{gathered}
$$

The solution of the linearized Navier-Stokes equations (11)(13) is quite difficult because the boundary condition (12) involves the rough wall with random inhomogeneities. Using a coordinate transformation $y \rightarrow y-\xi(x)$, we can reduce the Navier-Stokes equation to an equivalent equation with the boundary condition on the perfect flat wall. However, this new equation, as a result of the transformation-driven change in derivatives, acquires several additional terms $\hat{V} \psi$ that involve the combinations of derivatives of $\psi$ and the random function $\xi(x)$. To deal with these terms, we find the explicit form of the Green's function with the proper boundary condition. Then the problem reduces to a rather transparent integral equation

$$
\begin{aligned}
\psi\left(k_{x}, y\right)= & \psi_{i n h}\left(k_{x}, y\right)+\int_{0}^{\infty} d y^{\prime} G\left(k_{x}, y, y^{\prime}\right) \\
& \times \int_{-\infty}^{\infty} \frac{d k_{x}^{\prime}}{2 \pi} \hat{V}\left(k_{x}-k_{x}^{\prime}, y^{\prime}\right) \psi\left(k_{x}^{\prime}, y^{\prime}\right) .
\end{aligned}
$$

This procedure and the explicit expressions for the unperturbed inhomogeneous solution $\psi_{i n h}\left(k_{x}, y\right)$, the perturbation $\hat{V}$, and the Green's function are given in Appendix A. In some sense, we shifted the difficulty from the boundary condition to the bulk equations with random sources of the special form. Note that Eq. (14) is still exact and, in principle, 
could be solved without the perturbation theory. The explicit form of Green's function is such that one can extract the main part of the solution in the closed form. Another possible approach to Eq. (14) is to apply the Wiener-Hermite functional expansion $[40,41]$.

Note, that, in contrast to the attempts of replacing the rough surfaces by an effective thin layer of bulk scatterers [28], our procedure of replacing the random surface roughness by an equivalent bulk contribution is exact. What is more, the roughness-driven contributions to the flows are expressed explicitly via the geometrical profile of the rough surface.

Here, we solve Eq. (14) by iterations as an expansion in the small parameter: $\psi=\psi_{0}+\epsilon \psi_{1}+\epsilon^{2} \psi_{2}+\cdots$ The first three terms for the stream function have the following forms:

$$
\begin{gathered}
\psi_{0}\left(k_{x}, y\right)=\frac{2 \pi}{i \lambda} \delta\left(k_{x}\right) \exp (i \lambda y), \\
\psi_{1}\left(k_{x}, y\right)=\xi\left(k_{x}\right)\left[e^{i \lambda y}+\frac{i \lambda}{s_{2}-s_{1}}\left(e^{s_{1} y}-e^{s_{2} y}\right)\right], \\
\left\langle\psi_{2}\left(k_{x}, y\right)\right\rangle=\delta\left(k_{x}\right) \int_{-\infty}^{\infty} d k_{x}^{\prime} \zeta\left(k_{x}^{\prime}\right) \\
\times\left[\frac{s_{1}+s_{2}}{i \lambda}\left(i \lambda e^{i \lambda y}+s_{1} e^{s_{1} y}-s_{2} e^{s_{2} y}\right)\right],
\end{gathered}
$$

where we exclude uninteresting constant terms and

$$
\begin{gathered}
s_{1}=-\left|k_{x}\right|, \quad s_{2}=\sqrt{k_{x}^{2}-i \Lambda^{2}} \equiv-\alpha+i \beta, \\
\alpha, \beta=\frac{1}{\sqrt{2}} \sqrt{\left(k_{x}^{4}+\Lambda^{4}\right)^{1 / 2} \pm k_{x}^{2}} \geqslant 0, \\
\lambda=e^{i \pi / 4} \Lambda .
\end{gathered}
$$

Since for further calculations we need only the expression for $\psi_{2}$ which is averaged over the random surface inhomogeneities, Eq. (17) gives only the compact expression for $\left\langle\psi_{2}\left(k_{x}, y\right)\right\rangle$.

These expressions for the stream function provide the roughness-driven corrections for the velocity and rate of energy dissipation (see Appendix A):

$$
\begin{gathered}
\left\langle v_{x}\right\rangle=\operatorname{Re}\left\{e^{i(\lambda y-\omega t)}\left[1+i \lambda \epsilon^{2} \ell_{1}\right]\right\},\left\langle v_{y}\right\rangle=0, \\
\ell_{1}=\int_{0}^{\infty} \frac{d k_{x}}{\pi} \zeta\left(k_{x}\right)\left\{s_{1}+s_{2}-i \lambda / 2\right\}, \\
Q=-\frac{\eta u_{0}^{2}}{2 R} \frac{\Lambda}{\sqrt{2}}\left[1+\epsilon^{2} \Lambda^{2} \ell_{2}\right], \\
\ell_{2}=\int_{0}^{\infty} \frac{d t}{\pi} \zeta(t \Lambda) \phi(t), \phi(t)=1-\sqrt{\left(1+t^{4}\right)^{1 / 2}-t^{2}} .
\end{gathered}
$$

The equation for the energy dissipation is averaged over both the surface roughness and the over period of oscillations. This expression coincides with the result of Ref. [35] obtained with the help of the Rayleigh perturbation method.

Stream function also allows one to find corrections to the roughness-driven friction force. These calculations should be done more carefully than for standard flat geometry: the friction force is parallel to the actual surface and, in the case of the oscillating rough wall, has both components $F_{x}$ and $F_{y}$. One should also take into account the $y$ component of velocity, which is absent in the case of flat geometry. A straightforward calculation for the averaged square of absolute value of dimensionless friction force gives

$$
\mathbf{F}=\frac{\eta u_{0}}{R} \mathbf{f}, \quad\left\langle\overline{f^{2}}\right\rangle=\frac{\Lambda^{2}}{2}\left[1+\frac{\epsilon^{2} \Lambda^{2}}{\pi} \int_{0}^{\infty} d t \zeta(\Lambda t) \phi^{2}(t)\right] .
$$

This expression is different from a simple experimental definition of the effective friction force $F_{e f f}=-Q / u_{0}$.

At low frequencies (large decay lengths, $\Lambda \ll 1$ ), Eqs. (19) and (21) for parameters $\ell_{1,2}$ reduce to

$$
\begin{gathered}
\ell_{1}=-2 \int_{0}^{\infty} \frac{d k_{x}}{\pi} \zeta\left(k_{x}\right) k_{x}+O(\Lambda), \\
\ell_{2}=1+O(\Lambda \ln \Lambda),
\end{gathered}
$$

and the equations for the velocity and attenuation acquire the following form:

$$
\begin{aligned}
\left\langle v_{x}\right\rangle= & \operatorname{Re}\left\{e ^ { i ( \lambda y - \omega t ) } \left[1-\Lambda e^{i 3 \pi / 4}\right.\right. \\
& \left.\left.\times \epsilon^{2}\left(2 \int_{0}^{\infty} \frac{d k_{x}}{\pi} \zeta\left(k_{x}\right) k_{x}+O(\Lambda)\right)\right]\right\}, \\
Q=- & \frac{\eta u_{0}^{2}}{2 R} \frac{\Lambda}{\sqrt{2}}\left[1+\Lambda^{2} \epsilon^{2}(1+O(\Lambda \ln \Lambda))\right] .
\end{aligned}
$$

The fact that the main term in $\ell_{2}$ is equal to 1 is due to our choice of the normalization of the correlation function in Eq. (5) as $\zeta(x=0)=1$ (see also Sec. IV).

In the opposite limit of high frequencies $\Lambda \gg 1$,

$$
Q \approx-\frac{\eta u_{0}^{2}}{2 R} \frac{\Lambda}{\sqrt{2}}\left[1+\frac{\epsilon^{2}}{2} \int_{0}^{\infty} \frac{d k_{x}}{\pi} \zeta\left(k_{x}\right) k_{x}^{2}\right] \equiv Q_{0}\left[1+\frac{\epsilon^{2}}{2}\left\langle\xi^{\prime}\right\rangle\right] .
$$

This result has a simple physical explanation. In this limit, the decay length is much smaller than correlation radius (size) of the wall inhomogeneities $R$. As a result, the dissipation occurs in a very narrow layer near the wall within which the wall can be considered as almost flat. Then the correction to dissipation stems simply from the increase in the surface area relatively to the flat boundary 


$$
Q \approx-\frac{\eta u_{0}^{2}}{2 R} \frac{\Lambda}{\sqrt{2}} \frac{1}{L^{2}} \oint d A=-\frac{\eta u_{0}^{2}}{2 R} \frac{\Lambda}{\sqrt{2}} \frac{1}{L} \int \sqrt{1+\epsilon^{2} \xi^{\prime}} d x
$$

Equation (26) is simply the combination of the first two terms in the Taylor expansion of Eq. (27) in small $\epsilon$.

In principle, it is possible to slightly modify our problem by considering a torsional quartz crystal oscillator with density $\rho_{s}$, and thickness $d$. If such a resonator has a rough solid-fluid interface, the frequency shift $\delta \omega$ of the resonance frequency $\Omega_{0}$ acquires an additional roughness-driven component that can be described within the above formalism and should be given by similar equations. Such a frequency shift for a transverse oscillator is [35]

$$
\begin{aligned}
\delta \omega= & -\frac{\eta}{\sqrt{2}} \frac{\Lambda}{R} \frac{1}{\rho_{s} d}\left\{1+\epsilon^{2} \Lambda \int_{0}^{\infty} \frac{d k_{x}}{\pi} \zeta\left(k_{x}\right)\right. \\
& \left.\times\left[\sqrt{\left(k_{x}^{4}+\Lambda^{4}\right)^{1 / 2}+k_{x}^{2}}-\Lambda+\sqrt{2} k_{x}\right]\right\},
\end{aligned}
$$

where $\phi(y)$ is given by Eq. (22). We do not want to dwell on this issue; our interest in focused mainly on the roughnessdriven corrections to the hydrodynamic flows and dissipation.

\section{EFFECTIVE STICK-SLIP BOUNDARY CONDITIONS}

The main objectives of this paper are to find when and to what extent the flows near random rough surface are equivalent to stick-slip motion with some effective stick-slip boundary conditions near flat surfaces,

$$
\operatorname{Re}\left\{v_{x}(x, 0, t)-\frac{\ell_{e f f}}{R} \frac{\partial v_{x}(x, 0, t)}{\partial y}\right\}=\operatorname{Re}\left(e^{-i \omega t}\right),
$$

where the effective stick-slip length $\ell_{\text {eff }}$, in order to simplify the applications of the results, is introduced with the proper dimensionality of length while all other variables are still dimensionless, Eq. (5). With this boundary condition on a flat wall, the velocity field is

$$
v_{x}(y, t)=\operatorname{Re}\left[\frac{e^{i(\lambda y-\omega t)}}{1-e^{i 3 \pi / 4} \Lambda \ell_{e f f} / R}\right] .
$$

Since the roughness-generated corrections for velocity are small, the comparison between Eq (29) and Eqs. (19) and (24) is possible only when $\Lambda \ell_{e f f} / R \ll 1$, i.e., only for relatively large decay lengths (low frequencies)

$$
v_{x}(y, t) \approx \operatorname{Re}\left[e^{i(\lambda y-\omega t)}\left(1+e^{i 3 \pi / 4} \Lambda \ell_{e f f} / R\right)\right] .
$$

In this case, the comparison with the roughness-driven correction for the velocity, Eq. (24), yields the following simple expression for the effective stick-slip length $\ell_{e f f}=R \epsilon^{2} \ell_{1}$ :

$$
\ell_{e f f}=-2 \frac{h^{2}}{R} \int_{0}^{\infty} \frac{d k_{x}}{\pi} \zeta\left(k_{x}\right) k_{x} .
$$

The negative sign in Eq. (31) means that the boundary roughness causes effective slow down of the liquid, i.e., the coefficient $\ell_{\text {eff }}$ (31) is the stick length rather than the slip length. In other words, there is an additional roughnessinduced friction.

Surprisingly, the effective boundary conditions (28) and (31), taken by itself, cannot emulate the roughness-driven attenuation (21). The reason is the presence normal flows near the boundary, $v_{y}(x)$, which are completely absent within the effective stick-slip description (28) and (29), in which $v_{y}=0$. The attempts to modify the boundary condition (28) so that to reproduce both the velocity and attenuation correctly by, for example, introducing a two-component or complex stick-slip length, fail. In order to emulate the correct behavior of liquid near a rough wall, one has not only to introduce the stick-slip length (28) and (31), but also to renormalize the viscosity near the wall as

$$
\eta_{e f f}(y)=\eta[1+\beta \delta(y)],
$$

where renormalization parameter $\beta$ is given by

$$
\beta \approx 2 \frac{h^{2}}{R^{2}}\left[\ell_{1}+\Lambda \ell_{2} / \sqrt{2}\right],
$$

or, in the case of small $\Lambda$,

$$
\beta \approx 2\left[\frac{\ell_{e f f}}{R}+\frac{\Lambda}{\sqrt{2}} \frac{h^{2}}{R^{2}}\right] .
$$

The effective boundary conditions (28), (31), (32), and (34) are the main results of this paper. These conditions allow one to replace the random rough boundary by an equivalent problem with the flat boundary and the effective stickslip length and renormalized viscosity. The necessity of the renormalization of the viscosity means that the rough surface slows the flow down and changes the attenuation. Usually, the slip boundary condition is understood in terms of the existence of a peculiar thin slip boundary layer with the thickness of the order of the mean free path and with the properties that are somewhat different from the rest of the liquid. In the case of the rough walls, one should not only introduce the effective stick-slip layer with the thickness that is determined by $\delta$ and $R$, but also to renormalize the viscosity in this layer explicitly. A simple physical model that clarifies the meaning of the effective parameters is given in Appendix B.

\section{COMPARISON FOR DIFFERENT TYPES OF RANDOM INHOMOGENEITIES}

In this section, we address the question whether it is possible to extract information on the properties of the rough surface from the frequency dependence of attenuation of transverse oscillations. Statistical properties of the random surface are described by the correlation function of surface inhomogeneities, $\Xi(X)=h^{2} \zeta(x), x=X / R$, Eq. (6). Experimentally, the correlation function can exhibit different types of long-range behavior and can assume various forms [39]. 


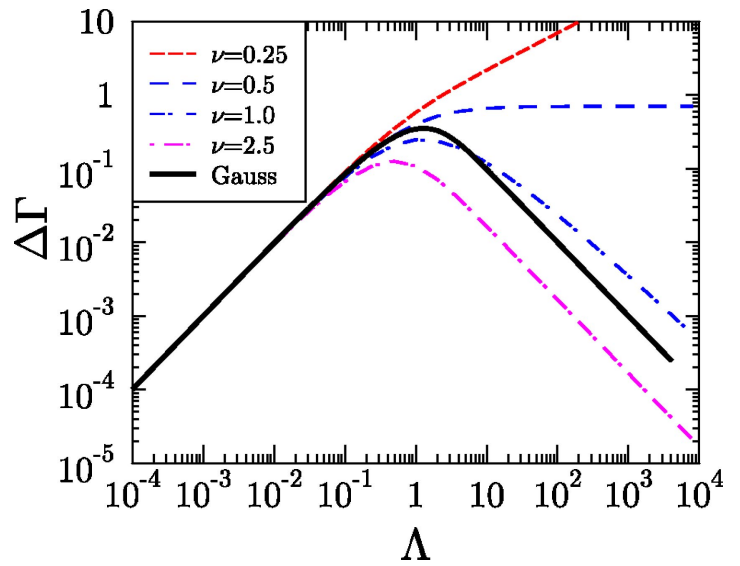

FIG. 2. Correction to the energy dissipation rate, $\Delta \Gamma$ as a function of the frequency parameter $\Lambda$ for Gaussian and $\nu$ correlators in $\log -\log$ scale.

$$
\begin{aligned}
\Delta \Gamma_{1} & \sim \int_{0}^{\Lambda} \frac{d k}{\left(1+k^{2}\right)^{\{\nu+1 / 2\}}}\left[\frac{k^{2}}{2 \Lambda}-\frac{k^{4}}{8 \Lambda^{3}}+\cdots\right] \\
& \sim \frac{1}{\Lambda}+O\left(\frac{1}{\Lambda^{2 \nu-1}}\right) .
\end{aligned}
$$

Thus, the energy dissipation rate for the correlators with the power-law power spectrum is determined by the value of the index $\nu$,

$$
\Delta \Gamma(\Lambda, \nu) \sim \begin{cases}\Lambda^{1-2 \nu}, & 0<\nu<1 \\ \Lambda^{-1}, & \nu>1\end{cases}
$$

Comparison of the asymptotic behavior of the function $\Delta \Gamma(\Lambda)$ for small and large $\Lambda$, Eqs. (38)-(40), indicates that this function should have a maximum at $\Lambda=\sqrt{2} R / \delta \sim 1$ except for the correlators with small $\nu$. In experiment, the position of this maximum on the frequency dependence of the attenuation can become a direct measurement of the correlation radius (size) of the surface inhomogeneities $R$.

The numerical results are summarized in Fig. 2 which presents the functions $\Delta \Gamma(\Lambda)$ for various correlators. $\mathrm{Nu}$ merical values of the position of the maximum for $\Delta \Gamma(\Lambda)$ for various correlation functions are presented in the Table I

The last column in the table describes the dimensionless roughness-driven stick-slip length $\ell_{1}=-\ell_{e f f} / \epsilon^{2} R$ for various correlators, Eq. (31) at small $\Lambda$,

$$
\ell_{1} \simeq 2 \int_{0}^{\infty} \frac{d k_{x}}{\pi} \zeta\left(k_{x}\right) k_{x} .
$$

\section{SUMMARY AND DISCUSSION}

In summary, we investigated the possibility of replacing a random rough surface by a set of effective stick-slip boundary conditions on an ideal flat surface. Such a replacement is highly desirable for analysis of experimental data and/or simplification of hydrodynamic computations for microchannels and nanochannels. The replacement turned out to be possible where the hydrodynamic decay length (the viscous wave penetration depth) is larger than the correlation radius (size) of random surface inhomogeneities. The effective boundary conditions contain two constants: the stick-slip length and the renormalization of viscosity near the boundary. The stick-slip length and the renormalization coefficient are expressed explicitly via the correlation function of surface inhomogeneities. The corresponding expressions are quite simple and can be easily used for analysis of experimental data or in hydrodynamic computations. The effective stick-slip length is negative. It means that the effective average hampering of the hydrodynamic flow by the rough surface (stick rather than slip motion). What is more, the renormalization coefficient for the viscosity is positive also pointing at additional resistance in the stick layer near the rough surface.

For a better understanding of the results, we presented a simple hydrodynamic model that illustrates our general hydrodynamic calculations.

In the process of the derivation of the effective boundary condition, we reduced the Navier-Stokes equation with the no-slip boundary condition on the random rough wall to the exactly equivalent closed integral equation with the homogeneous boundary condition on the ideal flat wall. All the information on the surface roughness is contained in the kernel of this integral equation. The equation can be solved by standard methods.

The effective boundary parameters were analyzed numerically for three classes of surface correlators including the Gaussian, power-law, and exponentially decaying correlators. The energy dissipation near the rough surface was calculated as a function of frequency for these types of the correlation functions. The position of maximum on the frequency dependence of the dissipation allows one to extract the correlation radius (characteristic size) of the surface inhomogeneities directly from, for example, experiments with torsional quartz oscillators.

In our particular hydrodynamic problem, the only hydrodynamic parameter with the dimensionality of length, which can be used for scaling of surface inhomogeneities, is the decay length $\delta$. In principle, in microchannels and nanochannels there is another scaling parameter-the channel width $L$. Therefore, the next obvious step should be the evaluation of the effective stick-slip length for ultrathin flow channels of the thickness $L$, for which $L$ is expected to gradually replace the decay length $\delta$ as the scaling parameter. The fact that our main result for the effective stick-slip length, Eq. (31), does not contain the decay length explicitly, gives hope that the same equation for the effective slip length will hold in finite channels as long as the channel width $L$ is larger than the amplitude and the correlation radius of the surface inhomogeneities $h$ and $R$.

In experiment, the measured slip length is a combination of the geometric, roughness-driven and physical, and molecular force-driven contributions. As it is shown above, the geometric contribution is always negative (stick rather than slip motion) and has the order of magnitude $-h^{2} / R$. On the other hand, the combined slip length can range between $1-100 \mathrm{~nm}[16,17,19,22-25]$. This slip length is routinely 
attributed to molecular forces. Since the roughness and molecular forces provide the contributions of the opposite signs, the slip length attributed to molecular forces in experiments with unknown surface roughness can be seriously underreported. The same concerns the renormalization of the viscosity near the surface and friction: the roughness-driven renormalization corresponds to the increase in viscosity and friction, while the molecular force driven renormalization, especially for hydrophobic solids, corresponds to a decrease in viscosity and friction.

Depending on the geometric parameters of the surface roughness, either one of the geometrical and physical contributions can dominate the overall slip. This transition from the force-driven contributions to the roughness-driven contributions has been recently observed in experiments with flows past the surfaces with different roughness but the same hydrophobic chemistry [20].

Quantitative analysis of experimental data requires the study of stick-slip motion which would take into account simultaneously the surface roughness and the molecular forces. For this, one would require calculation of the roughness-driven contribution in microchannels when the boundary condition on ideal walls corresponds to a partial, molecular force-driven slip. When the roughness-driven and molecular force-driven contributions to the surface slip and viscosity are independent, one would expect that the corresponding transport coefficients obey the Matthiessen's rule. Unfortunately, this is not always so: recent study of flow of particles with large mean free path through microchannels with random rough walls revealed large classical interference between bulk and wall scattering which resulted in the violation of the Matthiessen's rule [43] for bulk and wall-driven transport coefficients. In the systems in which the source of slip is not the large free path, but nonwetting of the walls, such interference may be caused by renormalization of molecular forces near walls due to the wall curvature.

\section{ACKNOWLEDGMENTS}

The work was supported by NSF Grant No. DMR0077266. We thank Dr. S. Granick (UIUC) for constructive comments and for providing us with several important references.

\section{APPENDIX A: SOLUTION OF THE NAVIER-STOKES EQUATION FOR FLUIDS RESTRICTED BY RANDOM ROUGH WALLS}

First, we assume that all the variables have the harmonic time dependence, $\exp (-i \omega t)$, transform the linearized Navier-Stokes equation (7) to the noninertial coordinate frame in which the wall is at rest,

$$
v_{x} \rightarrow v_{x}-\exp (-i \omega t), \quad x \rightarrow x-\int \exp (-i \omega t) d t
$$

and introduce the stream function $\psi(x, y)$ as

$$
v_{x}=\frac{\partial \psi}{\partial y}, \quad v_{y}=-\frac{\partial \psi}{\partial x} .
$$

In this reference frame, the Navier-Stokes equation (7) and the boundary condition (3) can be rewritten as the following equation for the stream function

$$
\begin{gathered}
-i \Lambda^{2} \nabla^{2} \psi-\nabla^{4} \psi=0, \\
\frac{\partial \psi(x, \epsilon \xi(x))}{\partial y}=1, \quad \frac{\partial \psi(x, \epsilon \xi(x))}{\partial x}=0, \\
\psi(x, \infty)=\text { const. }
\end{gathered}
$$

The difficulty in solving Eqs. (A2)-(A4) originates from the presence of a random function $\xi(x)$ in the boundary condition. The next step is the coordinate transformation

$$
x \rightarrow x, \quad y \rightarrow y-\epsilon \xi(x),
$$

that flattens the wall, making the boundary condition (12) simple,

$$
\frac{\partial \psi(x, 0)}{\partial y}=1, \quad \frac{\partial \psi(x, 0)}{\partial x}=\epsilon \xi_{x}(x), \quad \psi(x, \infty)=\text { const. }
$$

The change in derivatives introduces the additional term $\hat{V}\left(\xi, \partial_{x}\right) \psi$ into the RHS of Eq. (A2),

$$
-i \Lambda^{2} \nabla^{2} \psi-\nabla^{4} \psi=\hat{V}\left(\xi, \partial_{x}\right) \psi,
$$

where

$$
\begin{gathered}
\hat{V}=\epsilon \hat{V}_{1}+\epsilon^{2} \hat{V}_{2}+\epsilon^{3} \hat{V}_{3}+\epsilon^{4} \hat{V}_{4}, \\
\hat{V}_{1} \psi=-\left(2 \lambda^{2} \xi_{x} \psi_{y x}+\lambda^{2} \xi_{x x} \psi_{y}+4 \xi_{x x x} \psi_{y x}+6 \xi_{x x} \psi_{y x x}\right. \\
\left.+\xi_{x x x x} \psi_{y}+2 \xi_{x x} \psi_{y y y}+4 \xi_{x} \psi_{y x x x}+4 \xi_{x} \psi_{y y y x}\right), \\
\hat{V}_{2} \psi=\left(\lambda^{2} \xi_{x}^{2} \psi_{y y}+4 \xi_{x x x} \xi_{x} \psi_{y y}+12 \xi_{x} \xi_{x x} \psi_{y y x}+6 \xi_{x}^{2} \psi_{y y x x}\right. \\
\left.+2 \xi_{x}^{2} \psi_{y y y y}+3 \xi_{x x}^{2} \psi_{y y}\right), \\
\hat{V}_{3} \psi=-2 \xi_{x}^{2}\left(3 \xi_{x x} \psi_{y y y}+2 \xi_{x} \psi_{y y y x}\right), \\
\hat{V}_{4} \psi=\xi_{x}^{4} \psi_{y y y y},
\end{gathered}
$$

and lower indices denote the differentiation of the functions $\xi$ and $\psi$.

The simplicity of boundary conditions in new coordinates allows us to find the Green's function $G\left(x-x^{\prime}, y, y^{\prime}\right)$ for the operator on the LHS of Eqs. (A2) and (A7). With the help of this Green's function, our initial problem with a boundary condition on the random rough surface reduces to the compact integral equation,

$$
\begin{aligned}
\psi\left(k_{x}, y\right)= & \psi_{i n h}\left(k_{x}, y\right)+\int_{0}^{\infty} d y^{\prime} G\left(k_{x}, y, y^{\prime}\right) \\
& \times \int_{-\infty}^{\infty} \frac{d k_{x}^{\prime}}{2 \pi} \hat{V}\left(k_{x}-k_{x}^{\prime}, y^{\prime}\right) \psi\left(k_{x}^{\prime}, y^{\prime}\right),
\end{aligned}
$$


where we performed the Fourier transformation in $x$ direction (in the new coordinate frame, the geometry of the boundary is independent of $x$ ), and

$$
\psi_{i n h}\left(k_{x}, y\right)=\int_{-\infty}^{\infty} d x e^{-i k_{x} x} \psi(x, y)
$$

is a solution of Eq. (A2) with $\hat{V}=0$ and boundary conditions (A3), (A4). With this definition of $\psi_{i n h}\left(k_{x}, y\right)$, the Green's function satisfies the homogeneous boundary conditions on the wall. Note, that Eq. (A9) is an exact equivalent of our initial problem with the random rough wall and, in principle, can be solved for an arbitrary function $\xi(x)$.

The function $\psi_{i n h}\left(k_{x}, y\right)$ is determined by the characteristic equation for the operator in LHS of Eq. (A2):

$$
k_{y}^{4}-\left(2 k_{x}^{2}-i \Lambda^{2}\right) k_{y}^{2}-\left(i k_{x}^{2} \Lambda^{2}-k_{x}^{4}\right)=0 .
$$

This equation has four solutions,

$$
\begin{gathered}
k_{y}= \pm s_{1}, \pm s_{2}, \\
s_{1}=-\left|k_{x}\right|, \quad s_{2}=\sqrt{k_{x}^{2}-i \Lambda^{2}} \equiv-\alpha+i \beta, \\
\alpha, \beta=\frac{1}{\sqrt{2}} \sqrt{\left(k_{x}^{4}+\Lambda^{4}\right)^{1 / 2} \pm k_{x}^{2}} \geqslant 0 .
\end{gathered}
$$

We are interested only in the functions $\psi_{i n h}\left(k_{x}, y\right)$ that decrease at $y \rightarrow \infty$. Therefore, the general solution of the homogeneous Eq. (A2) with the boundary condition (A3) has the form

$$
\psi_{i n h}\left(k_{x}, y\right)=\frac{2 \pi}{i \lambda} \delta\left(k_{x}\right)\left[e^{i \lambda y}-1\right]+\frac{\epsilon \xi\left(k_{x}\right)}{s_{2}-s_{1}}\left[s_{2} e^{s_{1} y}-s_{1} e^{s_{2} y}\right]
$$

and contains the contribution without $\epsilon, \psi_{0}\left(k_{x}, y\right)$, and the term linear in $\epsilon$. Similar calculations yields the Green's function

$$
\begin{aligned}
G\left(k_{x}, y, y^{\prime}\right)= & \frac{1}{2 i \Lambda^{2}}\left[\frac{1}{s_{2}}\left(e^{s_{2}\left|y-y^{\prime}\right|}-e^{s_{2}\left(y+y^{\prime}\right)}\right)\right. \\
& \left.-\frac{1}{s_{1}}\left(e^{s_{1}\left|y-y^{\prime}\right|}-e^{s_{1}\left(y+y^{\prime}\right)}\right)\right] \\
& \times+\frac{1}{i \Lambda^{2}\left(s_{2}-s_{1}\right)}\left[e^{s_{1}\left(y+y^{\prime}\right)}+e^{s_{2}\left(y+y^{\prime}\right)}\right. \\
& \left.-e^{s_{1} y+s_{2} y^{\prime}}-e^{s_{1} y^{\prime}+s_{2} y}\right] .
\end{aligned}
$$

Note that the last result can be also obtained by noticing that our Green's function is proportional to the difference between the Green's functions for the two-dimensional Laplace and Helmholtz equations with the same boundary conditions:

$$
G\left(\mathbf{r}, \mathbf{r}^{\prime}\right)=\lambda^{-2}\left(G_{L}-G_{H}\right),
$$

$$
G_{L}\left(\mathbf{r}, \mathbf{r}^{\prime}\right)=-\frac{1}{2 \pi} \ln \left(R_{S} / R_{I}\right)
$$

$$
\begin{aligned}
G_{2}\left(\mathbf{r}, \mathbf{r}^{\prime}\right) & =\frac{i}{4}\left[H_{0}^{(1)}\left(\lambda R_{S}\right)-H_{0}^{(1)}\left(\lambda R_{I}\right)\right], \\
R_{S, I} & =\sqrt{\left(x-x^{\prime}\right)^{2}+\left(y \mp y^{\prime}\right)^{2}} .
\end{aligned}
$$

In our case of slight roughness, it is sufficient to find only the first three terms of the expansion of the stream function $\psi$, Eq. (A9), in powers of the small parameter $\epsilon, \psi=\psi_{0}$ $+\epsilon \psi_{1}+\epsilon^{2} \psi_{2}+\cdots$. Since all the terms in the operator $\hat{V}$ contain $\epsilon$, the only part of $\psi$ without $\epsilon$ is the first term in Eq. (A13) for $\psi_{i n h}$,

$$
\psi_{0}\left(k_{x}, y\right)=\frac{2 \pi}{i \lambda} \delta\left(k_{x}\right)[\exp (i \lambda y)-1]
$$

The first-order term in $\psi$ contains the remaining part of $\psi_{i n h}$ and the first order term in the integral (A9) with

$$
\hat{V}_{1}\left(k_{x}, \partial_{y}\right) \psi_{0}=-\xi\left(k_{x}\right)\left[k_{x}^{2} \lambda^{2}+k_{x}^{4}\right] e^{i \lambda y} .
$$

Integration gives

$$
\psi_{1}\left(k_{x,}, y\right)=\xi\left(k_{x}\right)\left[e^{i \lambda y}+\frac{i \lambda}{s_{2}-s_{1}}\left(e^{s_{1} y}-e^{s_{2} y}\right)\right]
$$

The calculation of the second-order term requires straightforward integration for much more cumbersome expressions. However, the general expression for $\psi_{2}$ is not required for further calculations; it is sufficient to have only the expression for $\psi_{2}$ averaged over the surface inhomogeneities, $\left\langle\psi_{2}\right\rangle$. The resulting expression for the stream function contains products of the derivatives of the surface profile $\xi^{(n)}(x)$. These products should be averaged over surface inhomogeneities using the definition of the correlation function $\zeta(x)$, Eqs. (6)

$$
\left\langle\xi^{(n)}(x) \xi^{(m)}\left(x^{\prime}\right)\right\rangle=(-1)^{m} \xi^{(n+m)}\left(x-x^{\prime}\right) .
$$

In the end, after substantial cancellations that accompany the averaging,

$$
\begin{aligned}
\left\langle\psi_{2}\left(k_{x}, y\right)\right\rangle= & \delta\left(k_{x}\right) \int_{-\infty}^{\infty} d k_{x}^{\prime} \zeta\left(k_{x}^{\prime}\right) \\
& \times\left[\frac{s_{1}+s_{2}}{i \lambda}\left(i \lambda e^{i \lambda y}+s_{1} e^{s_{1} y}-s_{2} e^{s_{2} y}\right)\right] .
\end{aligned}
$$

Reversing the coordinate transformation of Eq. (A5) and performing the related reexpansion in $\epsilon$, 


$$
\begin{aligned}
\left\langle v_{x}\left(k_{x}, y-\epsilon \xi\right)\right\rangle \simeq & v_{x}^{(0)}(y) \delta\left(k_{x}\right) \\
& +\epsilon^{2}\left[\left\langle v_{x}^{(2)}\left(k_{x}, y\right)\right\rangle-\left\langle\xi \frac{\partial}{\partial y} v_{x}^{(1)}\left(k_{x}, y\right)\right\rangle\right. \\
& \left.+\left\langle\frac{\xi^{2}}{2}\right\rangle \frac{\partial^{2}}{\partial y^{2}} v_{x}^{(0)}(y) \delta\left(k_{x}\right)\right]
\end{aligned}
$$

we get for the average velocity

$$
\begin{aligned}
\left\langle v_{x}(x, y)\right\rangle= & \exp (i \lambda y)\left[1+i \lambda \varepsilon^{2} \int_{0}^{\infty} \frac{d k_{x}}{\pi} \zeta\left(k_{x}\right)\right. \\
& \left.\times\left\{s_{1}+s_{2}-i \lambda / 2\right\}\right]
\end{aligned}
$$

where $s_{1}, s_{2}$ are given by Eq. (A12).

The above equations for the stream function and velocity allow one to calculate the roughness-driven correction to the dissipation of energy and effective friction.

Time average of the bulk dissipation per unit area of the wall can be expressed via the stream function $\psi$ as

$$
\begin{aligned}
Q & =-\frac{\eta u_{0}^{2}}{2 R} \frac{1}{A} \int d V\left\langle\overline{\left(\frac{\partial v_{i}}{\partial x_{k}}+\frac{\partial v_{k}}{\partial x_{i}}\right)^{2}}\right\rangle \\
& =-\frac{\eta u_{0}^{2}}{R} \frac{1}{A} \int d V\left\langle 4 \overline{\Psi_{x y}^{2}}+\overline{\left(\Psi_{y y}-\Psi_{x x}\right)^{2}}\right\rangle,
\end{aligned}
$$

where $\Psi(\mathbf{r}, t)=\operatorname{Re}\left[\psi(\mathbf{r}) e^{-i \omega t}\right]$, an overline denotes the time average over the period of oscillations and $\langle\ldots\rangle$ stands for the statistical average over the random surface inhomogeneities. The time average $\overline{\Psi_{i k}^{2}}=\frac{1}{2} \psi_{i k} \psi_{i k}^{*}$.

After the coordinate transformation (A5), the attenuation up to the second order term in $\epsilon$ reduces to

$$
\begin{gathered}
Q=-\frac{\eta u_{0}^{2}}{2 R} \int_{0}^{\infty} d y\left[Q^{(0)}+\epsilon^{2} Q^{(2)}\right], \\
Q^{(0)}=\left|\psi_{y y}^{(0)}\right|^{2}=\Lambda^{2} e^{-\sqrt{2} \Lambda y}, \\
Q^{(2)}=\left\langle\left|\psi_{y y}^{(1)}+\xi_{x x} \psi_{y}^{(0)}-\psi_{x x}^{(1)}\right|^{2}+2\left|\xi_{x} \psi_{y y}^{(0)}-\psi_{x y}^{(1)}\right|^{2}+2\left|\psi_{x y}^{(1)}\right|^{2}\right. \\
\left.+2 \operatorname{Re}\left[\psi_{y y}^{(0)} *\left(\psi_{y y}^{(2)}+\xi_{x x} \psi_{y}^{(1)}\right)\right]\right\rangle .
\end{gathered}
$$

Finally, we get

$$
\begin{aligned}
Q= & -\frac{\eta u_{0}^{2}}{2 R} \frac{\Lambda}{\sqrt{2}}\left\{1+\epsilon^{2} \Lambda \int \frac{d k_{x}}{2 \pi} \zeta\left(k_{x}\right)\right. \\
& \left.\times\left[\Lambda-\sqrt{\left(k_{x}^{4}+\Lambda^{4}\right)^{1 / 2}-k_{x}^{2}}\right]\right\} .
\end{aligned}
$$
[34]

The friction force acting on the area unit of the surface is

$$
\mathbf{F}=\frac{\eta u_{0}}{R} \mathbf{f}, \quad f_{i}=-\pi_{i k} n_{k}
$$

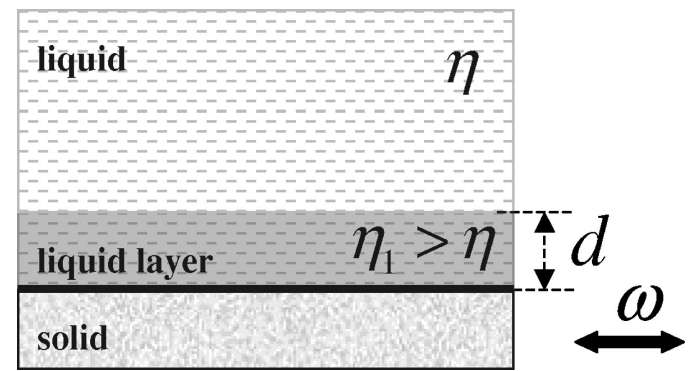

FIG. 3. Schematic geometry of the problem.

$$
\pi_{i k}=\left(\frac{\partial v_{i}}{\partial x_{k}}+\frac{\partial v_{k}}{\partial x_{i}}\right)_{y=\epsilon \xi}, \quad \mathbf{n}=\frac{1}{\sqrt{1+\epsilon^{2} \xi_{x}^{2}}}\left(\begin{array}{c}
\epsilon \xi_{x} \\
-1
\end{array}\right) .
$$

Here, $\mathbf{n}$ is the unit vector normal to the surface and directed out of the liquid. The square of the absolute value of this force is

$$
f^{2}=f_{x}^{2}+f_{y}^{2}=\pi_{x y}^{2}+\frac{\pi_{y y}^{2}+\epsilon^{2} \xi^{\prime 2} \pi_{x x}^{2}}{1+\epsilon^{2} \xi^{\prime 2}}
$$

or, via the stream function,

$$
f^{2}=\left.\left[\left(\psi_{y y}-\psi_{x x}\right)^{2}+4 \psi_{x y}^{2}\right]\right|_{y=\varepsilon \xi}
$$

In new coordinates (A5), this expression reduces to

$$
\left\langle f^{2}\right\rangle=\left\langle\left(1+2 \epsilon^{2} \xi_{x}^{2}\right) \psi_{y y}^{2}(x, 0)\right\rangle .
$$

After separating the real and imaginary parts and expanding in $\epsilon$, we finally get

$$
\left\langle f^{2}\right\rangle=\frac{\Lambda^{2}}{2}\left(1+\epsilon^{2} \int_{0}^{\infty} \frac{d k_{x}}{\pi} \zeta\left(k_{x}\right)\left[\Lambda-\sqrt{\left(k_{x}^{4}+\Lambda^{4}\right)^{1 / 2}-k_{x}^{2}}\right]^{2}\right) .
$$

Note that in this problem the friction force introduced by Eq. (A23) does not determine, after integration over the surface, the full energy dissipation. In the case of inhomogeneous rough boundaries there is an additional dissipative contribution related to the term with pressure, $P n_{i}$, in the expression for the full force acting on the unit area of the surface. If one defines the friction force not via the stress tensor, Eq. (A23), but assumes the experimental definition according to $F=-Q / u_{0}$, then the roughness-driven correction to the friction force will be given by Eq. (A22) rather than by Eq. (A27). Another anomaly of this problem is that one should always take into account both components of the friction force.

\section{APPENDIX B: TWO-LAYER MODEL}

The necessity of using two boundary parameters instead of a single stick-slip length can be illustrated by the following simple model. Let us consider tangential oscillations of viscous liquid which is separated from a solid substrate by a layer of another liquid with a slightly higher viscosity $\eta_{1}$ 
$>\eta$ and the same density (see Fig. 3). In effect, we model a rough surface by a layer of viscous liquid with somewhat different properties than in the bulk. The model has two parameters: the thickness of the layer $d$ and dimensionless ratio $\gamma$,

$$
\gamma=\frac{\eta \lambda}{\eta_{1} \lambda_{1}} \equiv \frac{\Lambda_{1}}{\Lambda} \lesssim 1 .
$$

Assuming that the velocity in both liquids is proportional to $\exp (-i \omega t)$, we get the following equations of motion:

$$
\begin{gathered}
-i \omega v_{1}-\nu_{1} \frac{d^{2} v_{1}}{d y^{2}}=0, \quad-i \omega v-\nu \frac{d^{2} v}{d y^{2}}=0, \\
v_{1}(0)=1, \quad v_{1}(d)=v(d), \\
\eta_{1} \frac{d v_{1}(d)}{d y}=\eta \frac{d v(d)}{d y} .
\end{gathered}
$$

The solution is

$$
\begin{gathered}
v_{1}(y)=A e^{i \lambda_{1}(y-d)}+B e^{-i \lambda_{1}(y-d)}, \\
v_{2}(y)=C e^{i \lambda y}
\end{gathered}
$$

with

$$
\begin{gathered}
A, B=C e^{i \lambda d} \frac{1 \pm \gamma}{2} \\
C=\frac{e^{-i \lambda d}}{\cos \left(\lambda_{1} d\right)-i \gamma \sin \left(\lambda_{1} d\right)} .
\end{gathered}
$$

Time average of the rate of the energy dissipation per unit consists of contributions from both liquids:

$$
\begin{gathered}
Q=Q_{I}+Q_{I I}, \\
Q_{I}=-\eta_{1} \int_{0}^{d} d y \overline{\left[\operatorname{Re}\left(\frac{\partial v_{1}}{\partial y} e^{-i \omega t}\right)\right]^{2}} \\
=-\left|C e^{i \lambda d}\right|^{2} \eta_{1} \Lambda_{1} \frac{1}{8 \sqrt{2}}\left[(1+\gamma)^{2}\left(e^{\sqrt{2} \lambda_{1} d}-1\right)\right. \\
\left.+(1-\gamma)^{2}\left(1-e^{-\sqrt{2} \lambda_{1} d}\right)+2\left(\gamma^{2}-1\right) \sin \left(\sqrt{2} \lambda_{1} d\right)\right],
\end{gathered}
$$

$$
\begin{aligned}
Q_{I I} & =-\eta \int_{d}^{\infty} d y \overline{\left[\operatorname{Re}\left(\frac{\partial v_{2}}{\partial y} e^{-i \omega t}\right)\right]^{2}} \\
& =-\left|C e^{i \lambda d}\right|^{2} \frac{\eta \Lambda}{2 \sqrt{2}} .
\end{aligned}
$$

If the thickness of the layer $d$ is smaller than the decay length $\delta, \lambda_{1} d \ll 1$,

$$
\begin{gathered}
C \rightarrow 1-e^{i 3 \pi / 4} \Lambda d\left(1-\gamma^{2}\right), \\
\left|C e^{i \lambda d}\right|^{2} \rightarrow 1-\sqrt{2} \Lambda d \gamma^{2}+\Lambda^{2} d^{2} \gamma^{4}, \\
v(y) \approx e^{i \lambda y}\left[1-i \lambda d\left(1-\gamma^{2}\right)\right] \\
-Q \approx \frac{\eta \Lambda}{2 \sqrt{2}}\left[1+\Lambda^{2} d^{2} \gamma^{2}\left(1-\gamma^{2}\right)\right] .
\end{gathered}
$$

Note that the condition $\lambda_{1} d \ll 1$ does not necessarily mean that the layer is very thin.

The last two equations show that in this limit

$$
v(y \geqslant d) \approx \operatorname{Re}\left\{u_{0} e^{i(\lambda y-\omega t)}\left[1-e^{i 3 \pi / 4} \Lambda d\left(1-\gamma^{2}\right)\right]\right\},
$$

$$
Q \approx \frac{\eta u_{0}^{2} \Lambda}{2 \sqrt{2}}\left[1+\Lambda^{2} d^{2} \gamma^{2}\left(1-\gamma^{2}\right)\right] .
$$

Comparison of Eqs. (B1) and (B2) with Eqs. ( 31)-(34) gives the mapping of the effective viscous layer model onto the problem with a rough surface,

$$
\begin{gathered}
-d\left(1-\gamma^{2}\right)=\ell_{e f f} / R \equiv \epsilon^{2} \ell_{1}, \\
d^{2} \gamma^{2}\left(1-\gamma^{2}\right)=\epsilon^{2} \ell_{2} .
\end{gathered}
$$

In this limit $d \ll \delta$, the contribution of the layer to the dissipation, $Q_{I}$, corresponds to the $\delta$-type renormalization of the viscosity in the effective boundary condition of Sec. III with renormalization parameter

$$
\beta=\epsilon^{2}\left[2 \ell_{1}+\sqrt{2} \Lambda \ell_{2}\right]
$$

given in Eq. (33).
[1] J.C. Maxwell, Scientific Papers (Dover, New York, 1953), Vol. 2, p. 704.

[2] M. Knudsen, Kinetic Theory of Gases (Methuen, London, 1950).

[3] A. Kundt, and E. Warburg, Ann. Phys. (Leipzig) 155, 337 (1875).

[4] P. Welander, Ark. Fys. 7, 507 (1954).

[5] D.R. Willis, Phys. Fluids, 5, 127 (1962).

[6] S. Albertoni, C. Cercigniani, and L. Gotusso, Phys. Fluids 6, 993 (1963).
[7] M.N. Kogan, Rarefied Gas Dynamics (Plenum, New York 1969).

[8] J.M. Parpia, and T.L. Rhodes, Phys. Rev. Lett. 51, 805 (1983).

[9] D.A. Ritchie, J. Saunders, and D.F. Brewer, Phys. Rev. Lett. 59, 465 (1987)

[10] J.E. Jaffe, J. Low Temp. Phys. 37, 567 (1979).

[11] H.H. Jensen et al., J. Low Temp. Phys. 41, 473 (1980).

[12] D. Einzel, H.H. Jensen, H. Smith, and P. Wolfle, J. Low Temp. Phys. 53, 695 (1983).

[13] H. Smith, Prog. Low Temp. Phys. 11, 75 (1987). 
[14] D. Einzel and J.M. Parpia, Phys. Rev. Lett. 58, 1937 (1987).

[15] K.D. Ivanova-Moser, and A.E. Meyerovich, J. Low Temp. Phys. 97, 55 (1994).

[16] A.L. Weisenhorn, P. Maivald, H.-J. Butt, and P.K. Hansma, Phys. Rev. B 45, 11226 (1992); E. Bonaccurso, M. Kappl, and H.-J. Butt, Phys. Rev. Lett. 88, 076103 (2002).

[17] O.I. Vinogradova, G.E. Yakubov, and H.-J. Butt, J. Chem. Phys. 114, 8124 (2001); R.G. Horn, O.I. Vinogradova, M.E. Mackay, and N. Phan-Thien, ibid. 112, 6424 (2000).

[18] J. Crassous, E. Charlaix, and J.-L. Loubet, Phys. Rev. Lett. 78, 2425 (1997).

[19] A. Carambassis, L.C. Jonker, P. Attard, and M.W. Rutland, Phys. Rev. Lett. 80, 5357 (1998); J.W.G. Tyrrell and P. Attard, ibid. 87, 176104 (2001).

[20] G. Reiter, A.L. Demirel, J. Peansky, L.L. Cai, and S. Granick, J. Chem. Phys. 101, 2606 (1994); A.L. Demirel and S. Granick, Phys. Rev. Lett. 77, 4330 (1996); J. Chem. Phys. 109, 6889 (1998); Y. Zhu and S. Granick, Phys. Rev. Lett. 88, 106102 (2002).

[21] F. Restagno, J. Crassous, C. Cottin-Bizonne, and E. Charlaix, Phys. Rev. E 65, 042301 (2002).

[22] V.S.J. Craig, C. Neto, and D.R.M. Williams, Phys. Rev. Lett. 87, 054504 (2001).

[23] K.B. Migler, H. Hervet, and L. Leger, Phys. Rev. Lett. 70, 287 (1993); R. Pit, H. Hervet, and L. Leger, ibid. 85, 980 (2000).

[24] L. Daikhin, E. Gileadi, V. Tsionsky, M. Urbakh, and G. Zilberman, Electrochim. Acta 45, 3615 (2000).

[25] O.I. Vinogradova, Int. J. Min. Process. 56, 31 (1999); Langmuir 11, 2213 (1995).
[26] J.F. Nye, Proc. R. Soc. London, Ser. A 311, 445 (1969); 315, 381 (1970).

[27] S. Richardson, J. Fluid Mech. 59, 707 (1973).

[28] K.M. Jansons, Phys. Fluids 31, 15 (1988).

[29] D.R. Berard, P. Attard, and G.N. Patey, J. Chem. Phys. 98, 7236 (1993); H. Shinto, K. Uranishi, M. Miyahara, and K. Higashitani, ibid. 116, 9500 (2002).

[30] G.E. Karniadakis and A. Beskok, Micro Flows: Fundamentals and Simulation (Springer, New York, 2001).

[31] D. Xiu and G.E. Karniadakis, Comput. Methods Appl. Mech. Eng. 191, 4927 (2002); J. Comp. Phys. (to be published).

[32] D. Einzel, P. Panzer, and M. Liu, Phys. Rev. Lett. 64, 2269 (1990).

[33] P. Panzer, M. Liu, and D. Einzel, Int. J. Mod. Phys. 6, 3251 (1992).

[34] L.D. Landau and E.M. Lifshitz, Fluid Mechanics, 2nd ed. (Pergamon, New York, 1987).

[35] M. Urbakh and L. Daikhin, Phys. Rev. B 49, 4866 (1994).

[36] L. Daikhin and M. Urbakh, Phys. Rev. E 49, 1424 (1994).

[37] M.G. Rozman, M. Urbakh, and J. Klafter, Phys. Rev. Lett. 77, 683 (1996).

[38] W.K.-H. Chu, ZAMP 47, 591 (1996); J. Phys.: Condens. Matter 12, 8065 (2000).

[39] J.A. Ogilvy, Theory of Wave Scattering from Random Surfaces (Adam Hilger, Bristol, 1991).

[40] N. Wiener, Nonlinear Problems in Random Theory (MIT Press, Boston, 1958).

[41] C. Eftimiu, J. Electromagn. Waves Appl. 4, 847 (1990).

[42] A.E. Meyerovich and I.V. Ponomarev, Phys. Rev. B 65, 155413 (2002).

[43] A.E. Meyerovich, Low Temp. Phys. 124, 461 (2001). 